INPLASY

PROTOCOL

To cite: Hang et al. Spleen Vessels Preserving versus Warshaw's Technique in Spleen Preserving Distal Pancreatectomy: A Systematic Review and Meta-analysis. Inplasy protocol 2021120108. doi:

10.37766/inplasy2021.12.0108

Received: 23 December 2021

Published: 24 December 2021

Corresponding author:

Li Kezhou

huaxipancreas@163.com

Author Affiliation:

Department of Pancreatic

Surgery, West China Hospital, Sichuan University, Chengdu, China.

Support: None.

Review Stage at time of this submission: Preliminary searches.

Conflicts of interest: None declared.

\section{Spleen Vessels Preserving versus Warshaw's Technique in Spleen Preserving Distal Pancreatectomy: A Systematic Review and Meta-analysis}

\author{
Hang, $\mathrm{H}^{1}$; Xiong, J2; Li, K³.
}

Review question / Objective: There exists now two established surgical procedures to preserve the spleen, including SVP-DP and WT, both of which are effective and safe ways for patients with benign and borderline malignant neoplasms in the pancreatic body and tail. The inconsistencies in outcomes between the two techniques still exist, thus we will compare clinical outcomes between patients who underwent SPDP with splenic vessel preservation (SVP) and Warshaw' technique(WT), and laparoscopic, robot-assisted and open surgery SPDP will all be considered.

Condition being studied: Spleen Vessels Preserving versus Warshaw's Technique in Spleen Preserving Distal Pancreatectomy. The research team comes from the Department of Pancreatic Surgery, West China Hospital, Sichuan University,Chengdu, China, and all the team members have perfect clinical experience in treating pancreatic neoplasms. Surgical outcomes of different surgical techniques will be analysed.

INPLASY registration number: This protocol was registered with the International Platform of Registered Systematic Review and Meta-Analysis Protocols (INPLASY) on 24 December 2021 and was last updated on 24 December 2021 (registration number INPLASY2021120108).

\section{INTRODUCTION}

Review question / Objective: There exists now two established surgical procedures to preserve the spleen, including SVP-DP and WT, both of which are effective and safe ways for patients with benign and borderline malignant neoplasms in the pancreatic body and tail. The inconsistencies in outcomes between the two techniques still exist, thus we will compare clinical outcomes between patients who underwent SPDP with splenic vessel preservation (SVP) and Warshaw' 
technique(WT), and laparoscopic, robotassisted and open surgery SPDP will all be considered.

Condition being studied: Spleen Vessels Preserving versus Warshaw's Technique in Spleen Preserving Distal Pancreatectomy. The research team comes from the Department of Pancreatic Surgery, West China Hospital, Sichuan University, Chengdu, China, and all the team members have perfect clinical experience in treating pancreatic neoplasms. Surgical outcomes of different surgical techniques will be analysed.

\section{METHODS}

Participant or population: Patients who underwent spleen preserving distal pancreatectomy.

Intervention: Patients who underwent spleen preserving distal pancreatectomy with spleen vessels preserving methods or Kimura's technique.

Comparator: Patients who underwent spleen preserving distal pancreatectomy with spleen vessels ligating methods or Warshaw's technique.

Study designs to be included: Controlled studies.

Eligibility criteria: SPDP performed on patients with benign or borderline malignant tumors in the distal pancreas.

Information sources: Major public medical and scientific databases including Pubmed, Embase, and Science Citation Index Expanded and The Cochrane Library.

Main outcome(s): Postoperative spleen infarction, perigastric/gastric varices, major complications (Clavien- Dindo III-V complications), clinical related postoperative pancreatic fistula (POPF, ISGPF grades $B$ and $C$ ) and reoperation.

Quality assessment / Risk of bias analysis: Newcastle-Ottawa Scale (NOS) will be used to assess the quality of each study, and we regard that scored $\geq 7$ as high-quality research. The potential publication bias of the selected studies will be tested by using a funnel plot.

Strategy of data synthesis: OR / mean difference (MD) with $95 \% \mathrm{Cl}$ will be calculated. A random-effects model or a fixed-effects mode will be chosen when significant heterogeneity or nonsignificant heterogeneity was not observed respectively.

Subgroup analysis: Subgroup analyses will be performed by respectively analyzing only high quality studies (NOS score $\geq 7$ ), studies in which operations were performed via LSPDP and studies with MIDP in which $n>50$.

Sensitivity analysis: Using Revman5. 4 software.

Country(ies) involved: China.

Keywords: pancreatic neoplasm, spleen preserving distal pancreatectomy, Kimura's procedure, Warshaw's technique.

Contributions of each author:

Author 1 - Hang Kuan.

Author 2 - Xiong Junjie.

Email: 474390090@qq.com

Author 3 - Li Kezhou.

Email: huaxipancreas@163.com 